\title{
Estrogen stimulates osteoprotegerin expression via the suppression of miR-145 expression in MG-63 cells
}

\author{
JUN JIA $^{1,2^{*}}$, HENGXING ZHOU ${ }^{1 *}$, XIANTIE ZENG ${ }^{2}$ and SHIQING FENG ${ }^{1}$ \\ ${ }^{1}$ Department of Orthopaedics, Tianjin Medical University General Hospital, Tianjin 300052; \\ ${ }^{2}$ Department of Surgery of Foot and Ankle, Tianjin Hospital, Tianjin 300211, P.R. China
}

Received December 1, 2015; Accepted December 12, 2016

DOI: $10.3892 / \mathrm{mmr} .2017 .6168$

\begin{abstract}
Osteoprotegerin (OPG) is implicated in the pathogenesis of postmenopausal osteoporosis, and other metabolic bone diseases caused by estrogen deficiency. Previous studies have demonstrated that estrogen may stimulate OPG expression in osteoblast cells at the transcriptional level; however, whether estrogen can regulate OPG expression at a post-transcriptional level remains elusive. The present study aimed to investigate the role of microRNA (miRNA) in estrogen-mediated OPG production in human osteoblast-like MG-63 cells. The results from ELISA, western blotting and reverse transcription-quantitative polymerase chain reaction (RT-qPCR) confirmed that estrogen may upregulate OPG expression. Mechanistic studies indicated that estrogen increased the activity of a luciferase reporter harboring the OPG 3'-untranslated region (3'-UTR). Bioinformatics analysis demonstrated that there is a potential targeting site in the OPG 3'-UTR for miRNA (miR)-145, which is associated with osteoblast differentiation. The results of an RT-qPCR suggested that estrogen suppressed miR-145 expression. In addition, dual-luciferase assay, RT-qPCR and western blot analysis indicated that miR-145 directly targets and negatively regulates OPG expression. Furthermore, transfection of cells with miR-145 mimics was able to partially inhibit the induction of OPG expression by estrogen, thus confirming the
\end{abstract}

Correspondence to: Professor Shiqing Feng, Department of Orthopaedics, Tianjin Medical University General Hospital, 154 Anshan Road, Heping, Tianjin 300052, P.R. China

E-mail: fengshiqing19@163.com

*Contributed equally

Abbreviations: OPG, osteoprotegerin; miRNA, microRNA; RANKL, receptor activator of nuclear factor- $\kappa \mathrm{B}$ ligand; RANK, receptor activator of nuclear factor- $\mathrm{kB}$; ER, estrogen receptor; 3'-UTR, 3'-untranslated region; E2, 17 $\beta$-estradiol; HRP, horseradish peroxidase; ASO-miR-145, 2-O-methyl-modified antisense oligonucleotide of miR-145

Key words: estrogen, osteoprotegerin, miR-145, post-transcriptional levels, MG-63 cells role of miR-145 in estrogen-mediated OPG induction. Taken together, the results of the present study demonstrated that estrogen may post-transcriptionally regulate OPG expression through suppression of miR-145 expression.

\section{Introduction}

Osteoporosis is a major public health problem characterized by low bone mass and impairment of microarchitecture, resulting in fragility fractures (1). For postmenopausal women, estrogen deficiency is one of the most common causes of osteoporosis, which results in an increase in the lifespan of osteoclasts and a concomitant decrease in osteoblast lifespan. These effects of estrogen deficiency on bone cells have been demonstrated to result in bone resorption over bone formation, leading to a sustained bone loss (2).

The mechanism underlying the action of estrogen has been widely studied $(3,4)$. Among the reported mechanisms responsible for estrogen action on bone cells, previous studies have focused on the role of the receptor activator of nuclear factor kappa B ligand/receptor activator of nuclear factor kappa B/osteoprotegerin (RANKL/RANK/OPG) regulatory system in the anti-resorptive action of estrogen $(5,6)$. RANKL, which is the final effector in osteoclastogenesis, is expressed on the surface of osteoblasts. It binds to its cellular receptor, RANK, on pre-osteoclasts and promotes their differentiation and activation (7). Eghbali-Fatourechi et al (8) reported that upregulation of RANKL in bone marrow cells is an important determinant of increased bone resorption induced by estrogen deficiency in postmenopausal women. OPG, which is a decoy receptor for RANKL, is a soluble glycoprotein secreted by various mesenchymal-derived cells, including osteoblasts and bone marrow stromal cells $(9,10)$. OPG reduces bone resorption by inhibiting osteoclast differentiation via suppression of RANKL binding to its functional receptor RANK $(11,12)$. Previous studies have demonstrated that estrogen can induce gene expression and protein synthesis of OPG in in vitro human and rat models (13-16), as well as in vivo studies in postmenopausal women (17). Estrogen stimulates OPG expression mainly at a transcriptional level through the estrogen receptor (ER), particularly ER $\alpha(13-15,18)$. Furthermore, an estrogen response element has been identified in the OPG promoter (19). However, whether OPG is regulated at a post-transcriptional level by estrogen remains unknown. 
MicroRNAs (miRNAs) are endogenous, small, noncoding RNAs, 21 to 23 nucleotides in length, which regulate gene expression post-transcriptionally, usually by binding to specific miRNA recognition sequences located in the 3'-untranslated regions (3'-UTRs) of target mRNAs, which finally leads to mRNA degradation and/or inhibition of translation (20). It has previously been demonstrated that miRNAs serve critical roles in regulating osteoblast differentiation and bone formation (21). For example, recent studies have identified that miRNA (miR)-145 regulates osteoblast differentiation by targeting transcription factors, core-binding factor subunit beta $(\mathrm{Cbfb})$ and osterix $(\mathrm{Osx})(22,23)$. Yang et al (24) indicated that miR-21 downregulation may contribute to the tumor necrosis factor- $\alpha$-induced inhibition of bone formation in estrogen deficiency-induced osteoporosis. Another report revealed that miR-503 was markedly reduced in circulating progenitors of osteoclasts from patients with postmenopausal osteoporosis, and that miR-503 inhibited bone resorption by directly targeting RANK (25). However, few miRNAs are known to be involved in bone metabolism by regulating OPG expression. miR-21 was recently reported to directly target and suppress OPG expression to promote the resorbing activity of mature osteoclasts (26). However, whether miRNAs are implicated in OPG induction by estrogen remains unclear.

The present study aimed to determine whether estrogen could regulate OPG expression at a post-transcriptional level via miRNA. The results of the present study demonstrated that estrogen stimulated OPG expression via suppression of miR-145 expression. These findings may enrich understanding regarding the molecular events underlying estrogen-mediated bone metabolism.

\section{Materials and methods}

Cell culture. Human osteoblast-like MG-63 cells were obtained from the Type Culture Collection of Chinese Academy of Sciences (Shanghai, China). Cells were cultured in RPMI-1640 medium (Gibco; Thermo Fisher Scientific, Inc., Waltham, MA, USA) supplemented with $10 \%$ fetal bovine serum (Gibco; Thermo Fisher Scientific, Inc.), $100 \mathrm{U} / \mathrm{ml}$ penicillin and $100 \mu \mathrm{g} / \mathrm{ml}$ streptomycin in a humidified incubator containing $5 \% \mathrm{CO}_{2}$ at $37^{\circ} \mathrm{C}$. For estrogen treatment, MG-63 cells in logarithmic growth phase were treated with various concentrations $(0,1,10$ and $100 \mathrm{nM})$ of $17 \beta$-estradiol (E2; Sigma-Aldrich; Merck Millipore, Darmstadt, Germany) for $48 \mathrm{~h}$ at $37^{\circ} \mathrm{C}$. Transfection was performed using Lipofectamine ${ }^{\circledR} 2000$ reagent (Invitrogen; Thermo Fisher Scientific, Inc.) according to the manufacturer's protocol. Cells were trypsinized with trypsin (cat. no. 25200056; Gibco; Thermo Fisher Scientific, Inc.), counted, and $5 \times 10^{5}$ cells/well were seeded on 6-well plates the day prior to transfection to ensure a suitable cell confluence (70\%) on the day of transfection. Then the cells were transfected with antisense oligonucleotide-miR-145 (ASO-miR-145) or ASO-negative control (NC), miRNA-145 mimic (mimic-145; analog of miR-145) or the negative control mimic (mimic-NC) (Shanghai GenePharma Co., Ltd., Shanghai, China), and then the cells were incubated in a humidified incubator containing $5 \% \mathrm{CO}_{2}$ at $37^{\circ} \mathrm{C}$ for $48 \mathrm{~h}$. The sequence used were listed in Table I.
Western blot analysis. MG63 cells stimulated by different estrogen doses $(0,1,10$ or $100 \mathrm{nM})$ for $48 \mathrm{~h}$ and cells treated with ASO-NC, ASO-miR-145, mimic-NC or mimic miR-145 for $48 \mathrm{~h}$ were used to detect OPG protein levels. Following E2 treatment or transfection, MG-63 cells were lysed with radioimmunoprecipitation assay buffer $(150 \mathrm{mM} \mathrm{NaCl}$, $1 \%$ Nonidet P-40, $1 \%$ Triton X-100, $1 \mathrm{mM} \mathrm{MgCl}_{2}, 0.1 \%$ SDS, $10 \mathrm{mM}$ Tris-HCl, $\mathrm{pH}$ 7.4) for $30 \mathrm{~min}$ before harvesting the protein. The protein concentrations were measured with the Bradford assay (Bio-Rad Laboratories, Inc., Hercules, CA, USA). An equal amount of protein $(30 \mu \mathrm{g})$ was resolved on a $10 \%$ SDS-polyacrylamide gel and was then transferred to a nitrocellulose membrane. Membranes were incubated with blocking buffer at room temperature (RT) for $2 \mathrm{~h}$, followed by incubation with specific primary antibodies against OPG (dilution, 1:200; cat. no. sc-71747; Santa Cruz Biotechnology, Inc., Dallas, TX, USA) or GAPDH (dilution 1:500; cat. no. sc-293335; Santa Cruz Biotechnology, Inc.) overnight at $4^{\circ} \mathrm{C}$. The membranes were then washed with TBST buffer (0.05\% Tween-20, $150 \mathrm{mM} \mathrm{NaCl}, 50 \mathrm{mM}$ Tris, $\mathrm{pH}$ 7.4) and incubated with the horseradish peroxidase (HRP)-conjugated secondary antibody (dilution, 1:3,000; cat. no. sc-2375; Santa Cruz Biotechnology, Inc.) for $1 \mathrm{~h}$ at RT. The protein of interest was visualized by enhanced chemiluminescence (Applygen Technologies, Inc., Beijing, China). LabWorks 4.0 image acquisition and analysis software (UVP, LLC, Upland, CA, USA) was used for densitometric analysis.

OPG ELISA. OPG protein secretion into the medium was determined in triplicate measurements using the OPG ELISA kit (cat. no. BV51101; IBL International; Tecan, Männedorf, Switzerland) according to the manufacturer's protocol. Briefly, MG63 cells was stimulated by different estrogen doses $(0,1,10$ or $100 \mathrm{nM})$ and the medium were collected following treatment for $48 \mathrm{~h}$, the collected medium was added to wells (96-well plate), following incubation at RT for $1 \mathrm{~h}$. Biotin-labeled antibodies from the ELISA kit were added to each well and were incubated at RT for $1 \mathrm{~h}$. After washing, streptavidin-HRP conjugate was added, followed by substrate solution and subsequent stop solution. Absorbance readings were measured at $450 \mathrm{~nm}$.

Reverse transcription-quantitative polymerase chain reaction $(R T-q P C R)$ assay. Total RNA was extracted using TRIzol reagent (Invitrogen; Thermo Fisher Scientific, Inc.) according to the manufacturer's protocol. RNA was reverse-transcribed to cDNA with oligo (dT) primers or a miR-145 specific primer using the Moloney murine leukemia virus reverse transcriptase (Promega Corporation, Madison, WI, USA). Total RNA $(2 \mu \mathrm{g})$ with the primer was incubated at $70^{\circ} \mathrm{C}$ for $5 \mathrm{~min}$, then added to the M-MLV 5X Reaction Buffer, dNTPs, RNasin (Promega Corporation), M-MLV RT enzyme (cat. no. M5301; Promega Corporation), and nuclease-free water to final volume of $25 \mu \mathrm{l}$, then was incubated for $1 \mathrm{~h}$ at $42^{\circ} \mathrm{C}$. RT-qPCR was performed with SYBR Premix EX Taq ${ }^{\mathrm{TM}}$ kit (Takara Bio, Inc., Otsu, Japan). $\beta$-actin was used as an endogenous control to normalize OPG expression levels; U6 small nuclear RNA was used an endogenous control to normalize miR-145 expression levels. The PCR reaction $(20 \mu \mathrm{l})$ system contain SYBR-Green premix $10 \mu \mathrm{l}$, cDNA $2 \mu \mathrm{l}$, forward primer $(10 \mu \mathrm{M}) 1 \mu \mathrm{l}$, reverse 
primer $(10 \mu \mathrm{M}) 1 \mu \mathrm{l}$ and $\mathrm{H}_{2} \mathrm{O} 6 \mu 1$. PCR cycles were as follows: $94^{\circ} \mathrm{C}$ for $3 \mathrm{~min}$ followed by 40 cycles of $94^{\circ} \mathrm{C}$ for $30 \mathrm{sec}, 56^{\circ} \mathrm{C}$ for $30 \mathrm{sec}$ and $72^{\circ} \mathrm{C}$ for $30 \mathrm{sec}$. All primers (GENEWIZ, South Plainfield, NJ, USA) used are listed in Table I. The relative fold-change in the transcripts were calculated using the $2^{-\Delta \Delta \mathrm{Cq}}$ method (27).

Plasmid construction. The 817-bp OPG 3'-UTR was amplified from MG-63 cDNA with the primers listed in Table I, and cloned into the dual-luciferase miRNA target expression vector pmirGLO (Promega Corporation) between the PmeI and XhoI sites. Mutations within the miR-145 binding sites were performed with the QuikChange Site-Directed Mutagenesis kit (Stratagene; Agilent Technologies, Inc., Santa Clara, CA, USA). All insertions were confirmed by DNA sequencing, performed by GENEWIZ. All primers (GENEWIZ) used are listed in Table I.

Luciferase reporter assay. For the luciferase assay, pmirGLO/OPG-3'-UTR or pmirGLO/OPG-3'-UTR mut or pmirGLO (Promega Corporation) along with mimic miR-145, mimic-NC, ASO-miR-145 or ASO-NC were transfected into MG-63 cells with Lipofectamine ${ }^{\circledR} 2000$ reagent (Invitrogen; Thermo Fisher Scientific, Inc.). 24 h following transfection, MG-63 cells were harvested and lysed with passive lysis buffer (Promega Corporation). Luciferase activity was measured using a dual luciferase reporter assay using a GloMax $^{\circledR}$ 20/20 Luminometer (Promega Corporation). The hRluc-neo was used as an internal control. The results were expressed as relative luciferase activity (LUC/Renilla LUC).

Statistical analysis. All experiments were performed at least three times. Data are presented as the mean \pm standard deviation. Statistical analysis was performed using SPSS version 15.0 (SPSS, Inc., Chicago, IL, USA) by conducting one-way analysis of variance. Comparisons between groups were analyzed with the Bonferroni post hoc test. $\mathrm{P}<0.05$ was considered to indicate a statistically significant difference.

\section{Results}

Estrogen stimulates $O P G$ expression. Previous studies have indicated that E2 can stimulate OPG expression in osteoblast cells (13-15). In order to confirm the stimulation of OPG by estrogen, the present study examined the effects of E2 on OPG expression in the human osteoblast cell line, MG-63. MG-63 cells were treated with various concentrations of E2 (1, 10 and $100 \mathrm{nM})$. The protein and mRNA expression levels of OPG were examined using ELISA, western blotting and RT-qPCR, respectively. As demonstrated in Fig. 1A-C, the protein expression levels of OPG in the culture medium and cytoplasm were significantly increased, even following treatment with the lowest E2 concentration (1 nM). The induction of OPG expression reached the highest levels following treatment with $10 \mathrm{nM}$ E2; however, $100 \mathrm{nM}$ was less potent. The effects of E2 on OPG mRNA indicated a similar pattern of results (Fig. 1D).

Estrogen post-transcriptionally stimulates $O P G$ expression via the 3'-UTR. It has been hypothesized that estrogen may stimulate OPG expression at the post-transcriptional level. In order to provide evidence supporting this hypothesis, the OPG 3'-UTR was inserted downstream of the luciferase reporter gene within the pmirGLO vector and transfected into MG-63 cells. The luciferase activities were examined following treatment with various doses of E2 for 48 h. As presented in Fig. 2, there was no response to E2 treatment for cells transfected with the reporter vector without any 3'-UTR. However, compared with untreated cells, the luciferase activities from the cells transfected with reporter vectors containing the OPG 3'-UTR were markedly stimulated by E2 in a dose-dependent manner (Fig. 2B, $\mathrm{P}<0.01$ ). These findings suggested that $\mathrm{E} 2$ regulation for OPG is specific and the OPG 3'-UTR is involved.

E2 regulation of $O P G$ involves $m i R-145$. Sequence analysis of the OPG 3'-UTR was performed using several web-based bioinformatics tools including TargetScan (http://www. targetscan.org/vert_71/), MicroCosm Targets (http://www.ebi .ac.uk/enright-srv/microcosm/htdocs/targets/v5/) and PicTar (www.pictar.org), and identified several potential binding sites for various miRNAs. The interest in miRNAs came from previously determined findings identifying that they are involved in bone homeostasis, and that they may selectively target the 3'-UTR of OPG mRNA. As a result, the present study identified a target site matched to the seed sequence of miR-145 (Fig. 3A), which was found to be involved in the regulation of osteoblast differentiation (26). To the best of our knowledge, the present study is the first to investigate the regulation of miR-145 by E2 in MG-63 cells. RT-qPCR analysis demonstrated that E2 treatment resulted in a significant decrease in miR-145 expression levels at concentrations of 10 and $100 \mathrm{nM}$ compared with the control group $(\mathrm{P}<0.01)$, and the effects of E2 reached a peak of $\sim 60 \%$ inhibition at $10 \mathrm{nM}$ (Fig. 3B).

The effects of miR-145 on OPG expression were subsequently investigated. Firstly, the effects of miR-145 on luciferase activity in a transient transfection system were measured using chemically synthesized miR-145 mimics and a 2-O-methyl-modified antisense oligonucleotide of miR-145 (ASO-miR-145) as an inhibitor of miR-145 (Fig. 4). Transfection of cells with miR-145 mimics induced a $~ 50 \%$ decrease in the activities of the reporter harboring OPG 3'-UTR. Conversely, inhibition of miR-145 with ASO-miR-145 resulted in an increase of $\sim 44 \%$ when compared with the ASO-NC-transfected cells (Fig. 4B). To determine the potential miR-145 target site responsible for regulation of the OPG 3'-UTR, a pmirGLO reporter vector containing the OPG 3'-UTR was constructed with a mutated miR-145 binding site (Fig. 3A). As expected, neither overexpression nor suppression of miR-145 had any effect on the luciferase activities of the reporter harboring mutated OPG 3'-UTR (Fig. 4C), thus suggesting that the predicted miR-145 binding site is functional.

In order to determine whether miR-145 also suppresses endogenous OPG at a post-transcriptional level, the effects of miR-145 on OPG mRNA and protein levels were analyzed using RT-qPCR and western blotting, respectively. As shown in Fig. 4D, overexpression of miR-145 led to a $\sim 35 \%$ reduction when compared with the negative control, and inhibition of miR-145 with the ASO resulted in a $\sim 66 \%$ increase in OPG mRNA compared with the negative control. In accordance with these results, overexpression of miR-145 reduced protein 
Table I. Primer sequences and oligomers used in the present study.

\begin{tabular}{|c|c|}
\hline Name & Sequence $\left(5^{\prime}-3^{\prime}\right)$ \\
\hline NC mimic & $\begin{array}{l}\text { UUCUCCGAACGUGUCACGUTT } \\
\text { ACGUGACACGUUCGGAVGGTT }\end{array}$ \\
\hline miR-145 mimic & $\begin{array}{l}\text { GUCCAGUUUUCCCAGGAAUCCCU } \\
\text { AGGGAUUCCUGGGAAAACUGGAC }\end{array}$ \\
\hline ASO-NC & GTGGATATTGTTGCCATCA \\
\hline ASO-miR145 & AGGGATTCCTGGGAAAACTGGAC \\
\hline miR-145-RT & $\begin{array}{l}\text { GTCGTATCCAGTGCAGGGTCCGAGGTGCACTGGA } \\
\text { TACGATGGGATTC }\end{array}$ \\
\hline U6-RT & $\begin{array}{l}\text { GTCGTATCCAGTGCAGGGTCCGAGGTATT } \\
\text { CGCACTGGATACGACAAAATATGGAAC }\end{array}$ \\
\hline miR-145-forward & TGCGGGTCCAGTTTTCCCAGGAATC \\
\hline Reverse & CCAGTGCAGGGTCCGAGGT \\
\hline U6-forward & TGCGGGTGCTCGCTTCGGCAGC \\
\hline Reverse & CCAGTGCAGGGTCCGAGGT \\
\hline OPG-sense & GGAAAGAAAGTGGGAGCAGAAGACAT \\
\hline OPG-antisense & CTGAGTGACAGTTTTGGGAAAGTGGT \\
\hline$\beta$-actin-sense & CGTGACATTAAGGAGAAGCTG \\
\hline$\beta$-actin-antisense & CTAGAAGCATTTGCGGTGGAC \\
\hline OPG-3'-UTR-sense & CGGAATTCACTGGAAATGGCCATTGAGCTGT \\
\hline OPG-3'-UTR-antisense & CGGCTCGAGTAAGGTACATTCAATTTCTCAG \\
\hline OPG-3'-UTR-sense-mut & AATTACGTGATGAATTACTGCAAGCAGTAATAAG \\
\hline OPG-3'-UTR-antisense-mut & CTTATTACTGCTTGCAGTAATTCATCACGTAATT \\
\hline
\end{tabular}

ASO, antisense oligonucleotide; miR-145, microRNA-145; mut, mutant; NC, negative control; OPG, osteprotegerin; RT, reverse transcription; UTR, untranslated region.

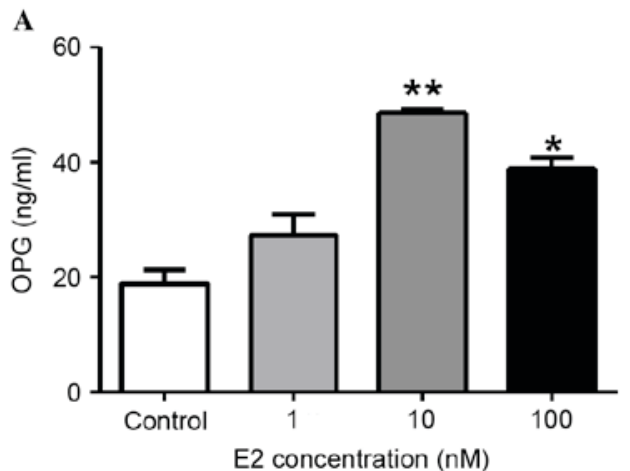

B
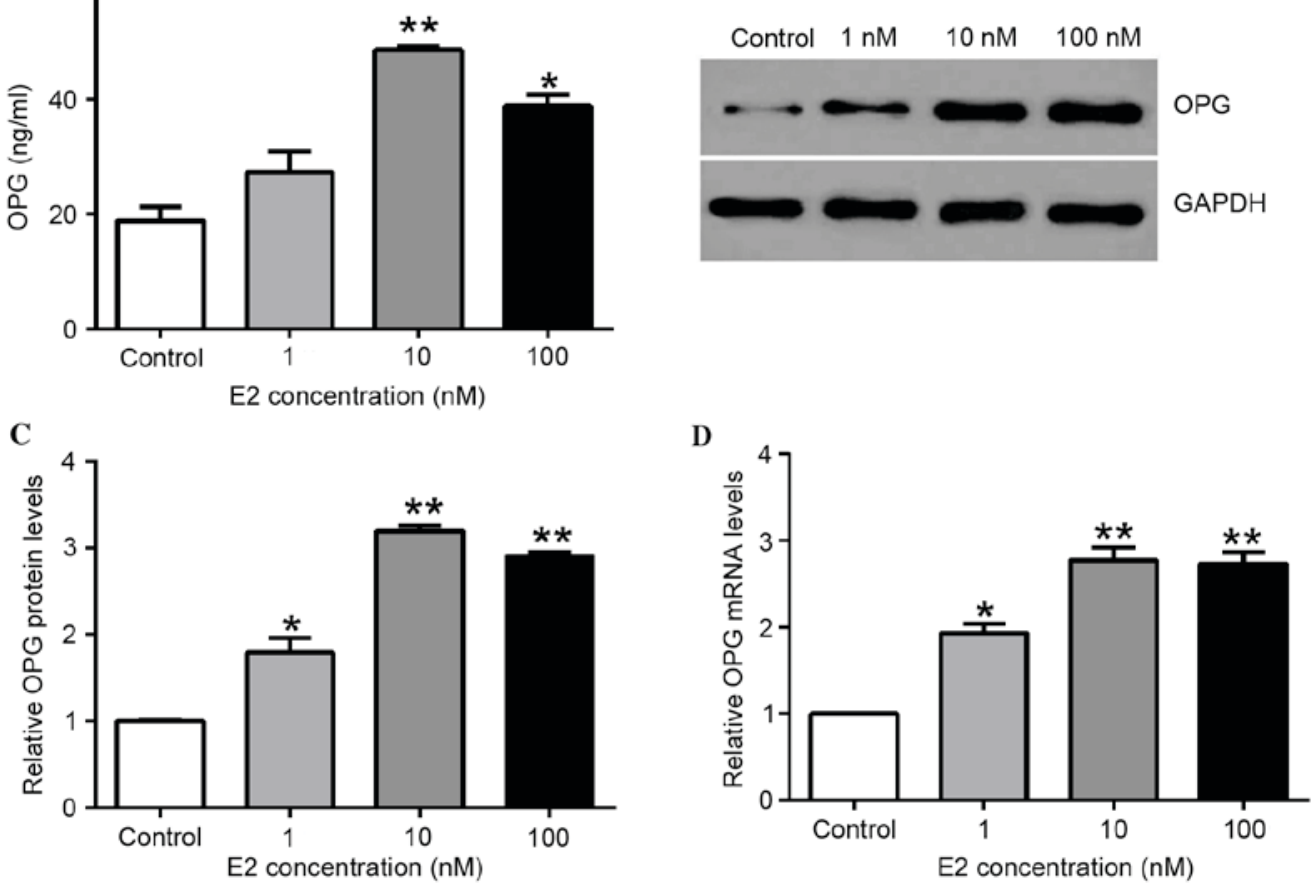

Figure 1.E2 stimulates OPG expression. MG-63 cells were treated with the indicated concentrations of E2 for $48 \mathrm{~h}$. (A) Levels of OPG protein secreted into the medium were measured by ELISA. (B and C) Levels of intracellular OPG protein were analyzed by western blot analysis and GAPDH was used for normalization. (D) OPG mRNA expression was detected by reverse transcription-quantitative polymerase chain reaction, and $\beta$-actin was used for normalization. ${ }^{*} \mathrm{P}<0.05,{ }^{* *} \mathrm{P}<0.01$ vs. control. E2, $17 \beta$-estradiol; OPG, osteoprotegerin. 

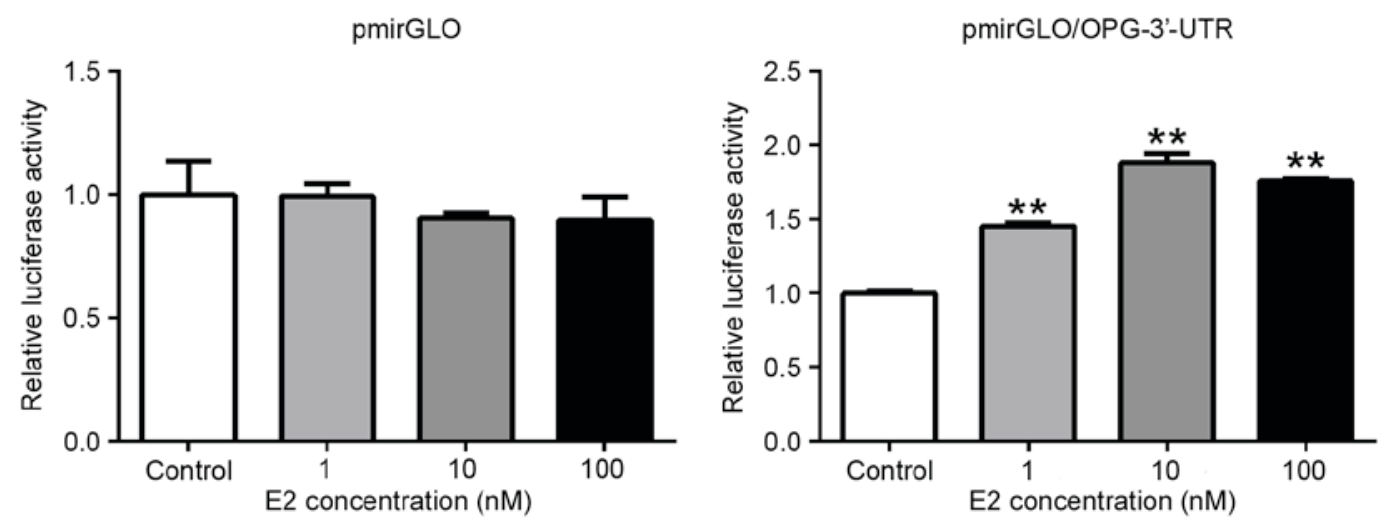

Figure 2. E2 stimulates OPG expression through its 3'-UTR. MG-63 cells were transfected with the control vector, pmirGLO, or the pmirGLO vector harboring the 3'-UTR of OPG. A total of $24 \mathrm{~h}$ post-transfection, the cells were treated with the indicated concentrations of E2 for $48 \mathrm{~h}$, subsequently the luciferase activities were examined by dual luciferase assay. The results were normalized to Renilla activity, and the untreated group was normalized as $1 .{ }^{* *} \mathrm{P}<0.01 \mathrm{vs}$. control. E2, 17ß-estradiol; mut, mutant; OPG, osteoprotegerin; UTR, untranslated region; wt, wild type.

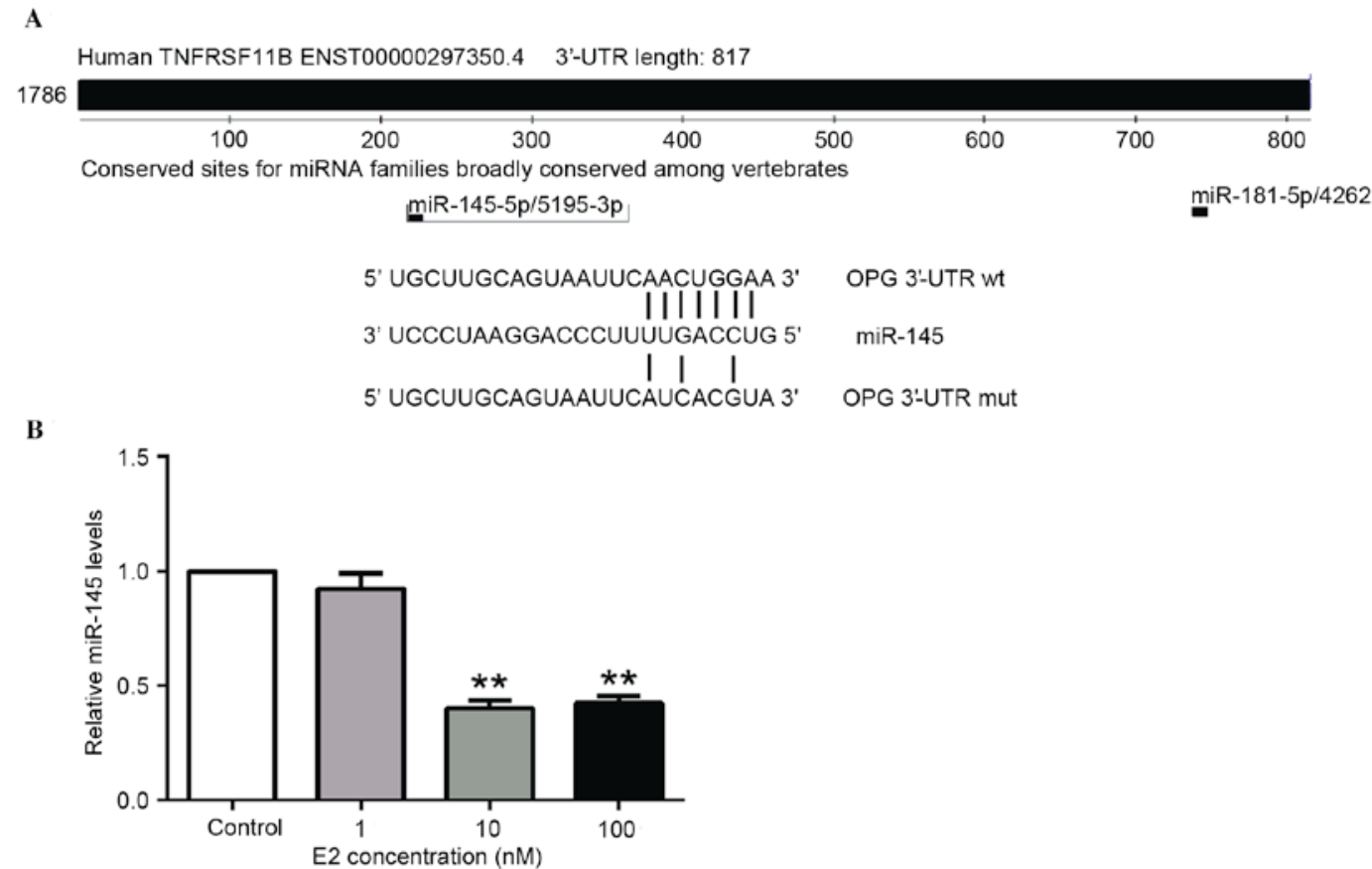

Figure 3. E2 decreases mature miR-145 levels. (A) miRNA target sites in the OPG 3'-UTR were analyzed by TargetScan. The potential miR-145 site is presented. (B) MG-63 cells were treated with the indicated concentrations of E2 for $48 \mathrm{~h}$, miR-145 levels were determined by reverse transcription-quantitative polymerase chain reaction. U6 was used as an internal control. ${ }^{* *} \mathrm{P}<0.01$ vs. control. E2, 17ß-estradiol; miR, microRNA; OPG, osteoprotegerin; TNFRSF11B, TNF receptor superfamily member 11b; UTR, untranslated region.

levels, whereas inhibition of miR-145 increased OPG protein levels (Fig. 4E and F).

Restoration of miR-145 compromises the induction of OPG by estrogen. To verify the role of miR-145 in estrogen-mediated OPG induction, MG-63 cells were transfected with miR-145 mimics prior to E2 treatment, and the level of OPG that was secreted into the medium, as well as OPG mRNA expression, were measured using ELISA and RT-qPCR, respectively. The results from the ELISA demonstrated that the induction of OPG by E2 treatment was partially counteracted post-transfection with miR-145 (Fig. 5A, P<0.01). RT-qPCR analysis of OPG mRNA indicated a similar result (Fig. 5B, P $<0.01$ ). Together, these results indicated that estrogen stimulated OPG expression at least partly through suppression of miR-145 expression.

\section{Discussion}

Previous studies have demonstrated that estrogen can stimulate OPG expression in human and rodent osteoblast cells, and the primary mechanism underlying this regulation is through the transcription factor ER $(13-15,18,19)$. The present study is the first, to the best of our knowledge, to indicate that estrogen can induce OPG expression at the post-transcriptional level via suppression of miR-145 expression in human osteoblast-like MG-63 cells. Considering the important role of OPG in regulating bone resorption, this result may aid in the understanding 
$\mathbf{A}$

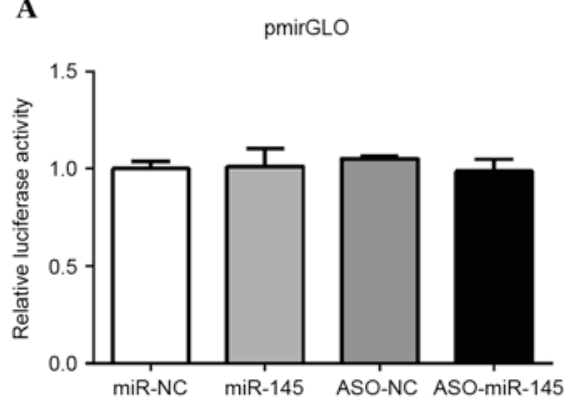

D

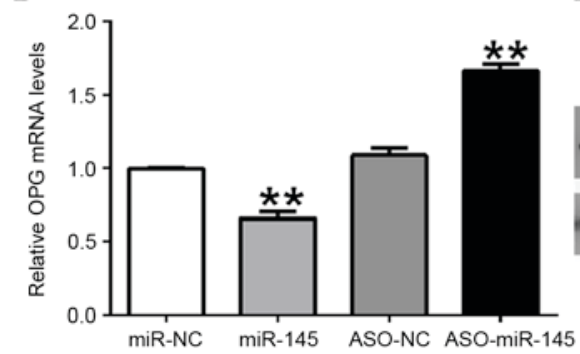

B

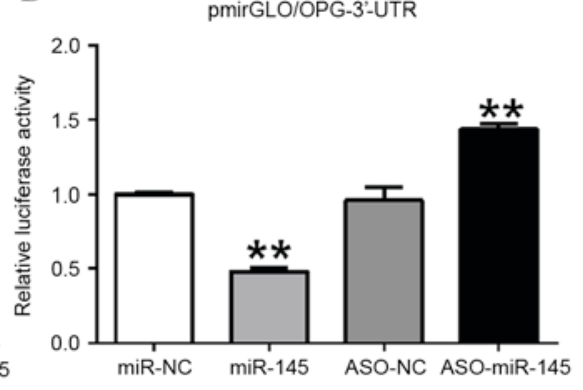

$\mathbf{E}$

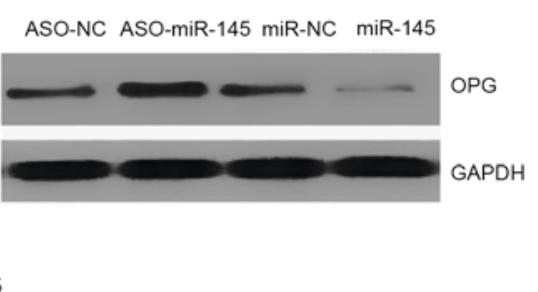

C

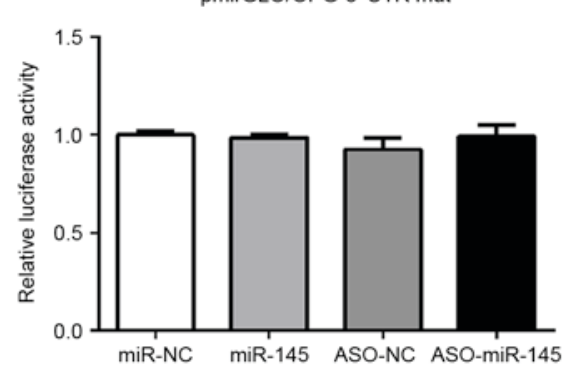

F

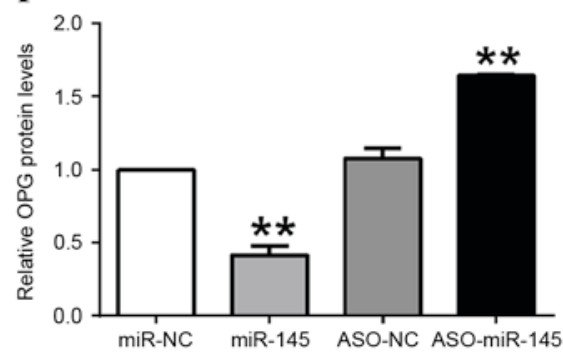

Figure 4. miR-145 targets the 3'-UTR of OPG and suppresses its expression. (A-C) miR-145 mimics or ASO-miR-145 were co-transfected with pmirGLO or pmirGLO vectors containing wt or mut 3'-UTRs of OPG into MG-63 cells. Following $48 \mathrm{~h}$, cells were harvested and assayed for dual luciferase activity. The results were normalized to Renilla activity. The effects of miR-145 on endogenous OPG expression were determined by (D) reverse transcription-quantitative polymerase chain reaction and (E and F) western blotting. The mRNA and protein expression levels of OPG were normalized to the levels of $\beta$-actin mRNA and GAPDH protein, respectively. ${ }^{* *} \mathrm{P}<0.01$ vs. control. ASO, antisense oligonucleotide; miR, microRNA; NC, negative control; OPG, osteoprotegerin; UTR, untranslated region.

A

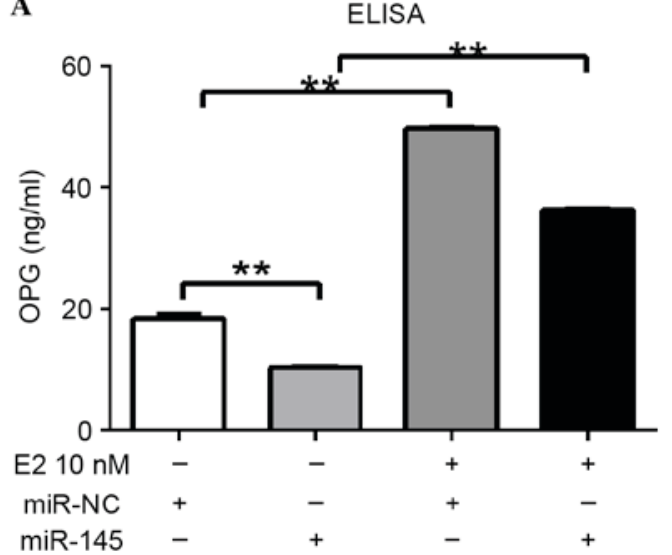

B

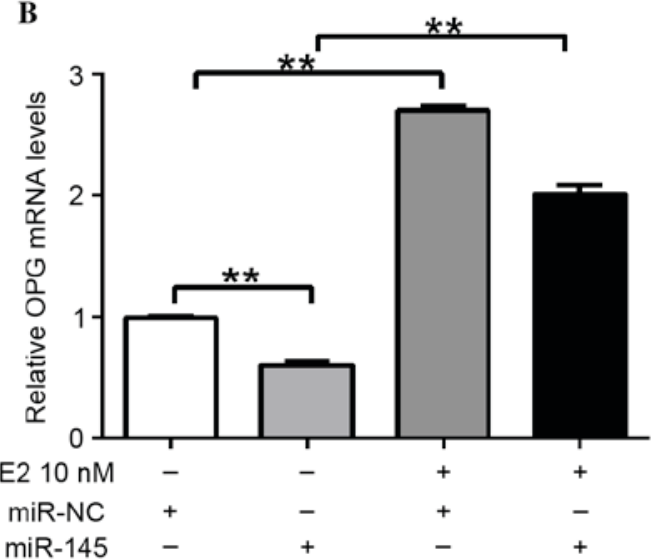

Figure 5. Overexpression of miR-145 compromises the induction of OPG by estrogen. MG-63 cells were transfected with miR-145 mimics or NC. A total of $24 \mathrm{~h}$ post-transfection, the cells were treated with $10 \mathrm{nM} \mathrm{E} 2$ for $48 \mathrm{~h}$, and the OPG protein levels secreted into the medium and OPG mRNA were measured by (A) ELISA and (B) reverse transcription-quantitative polymerase chain reaction. The mRNA expression levels of OPG were normalized to the mRNA expression levels of $\beta$-actin. ${ }^{* *} \mathrm{P}<0.01$ vs. control. E2, 17 $\beta$-estradiol; miR, microRNA; NC, negative control; OPG, osteoprotegerin.

of the molecular mechanism underlying estrogen therapy for osteoporosis.

The results of the present study indicated that estrogen at a concentration of $10 \mathrm{nM}$ can stimulate OPG expression by $\sim 3$-fold in human osteoblast-like MG-63 cells, which is consistent with the results from the human osteoblast cell lines and a mouse stromal cell line, ST-2 (13-15). However, estrogen at a higher concentration $(100 \mathrm{nM})$ did not exhibit a more potent stimulation on OPG expression, this may be limited by the presence of its receptor $\mathrm{ER} \alpha, \mathrm{ER} \beta$ in the nucleus and/or the membrane-associated receptor. This may be because there is a more complicated response following higher doses of estrogen treatment, which neutralizes its induction on OPG expression. The specific explanation requires further investigation.

It has been reported that estrogen activates its intracellular signaling via binding with the classic steroid receptors (ER $\alpha$ and ER $\beta$ ) or G-protein-coupled receptors (28). In human and rodent osteoblast cells, estrogen stimulates OPG expression in an ER-dependent manner $(13,14)$. Conversely, estrogen suppresses OPG production in MCF-7 breast cancer cell and in peripheral blood mononuclear cells $(29,30)$, and ER $\alpha$ mediated the suppression of OPG in MCF-7 cells (29). A previous report demonstrated that there is an estrogen response element within the OPG gene promoter (19), which suggested the direct 
regulation of estrogen on OPG gene transcription through ER. In human dental pulp cells, an ERK inhibitor significantly increased estradiol-induced OPG expression and a p38 inhibitor significantly attenuated this expression (16). All these findings indicated that the regulation of estrogen on OPG expression is complex and may occur at both a transcriptional and post-transcriptional level. For the important regulatory role of miRNA at a post-transcriptional level, the present study explored the possibility of miRNA in estrogen-mediated OPG induction. To identify the regulatory elements that are responsible for E2-mediated OPG induction, bioinformatics analysis was performed on the OPG 3'-UTR sequence, and the results indicated that there are several potential miRNA binding sites within the 3'-UTR of OPG. In combination with the reported function of miR-145, miR-145 was selected for further investigation. The results of the present study suggested that miR-145 was decreased by estrogen in a dose-dependent manner and that miR-145 suppressed OPG expression by directly binding to its 3'-UTR, which implicated its important role in regulating bone resorption. It has been reported that estrogens regulate miRNA transcription through multiple pathways (31-33). A previous study reported that estrogen upregulates OPG expression in an ER $\alpha$-dependent manner. Whether ER was responsible for the suppression of miR-145 by estrogen will be investigated in future studies.

miR-145 is a miRNA that is widely reported to be dysregulated in various types of human cancer, including breast, prostate, colon and lung cancer (34). In addition, miR-145 has been reported to be downregulated in osteosarcoma cells and tissues, and serves an important role in bone cell function $(35,36)$. miR-145 previously inhibited cell proliferation, migration, and invasive abilities of MG-63 cells by targeting Rho-associated protein kinase 1 (36). Furthermore, miR-145 served an important role in suppressing osteoblastic differentiation by targeting Osx and Cbfb, two transcription factors that are essential for bone formation $(22,23)$. The present study is the first, to the best of our knowledge, to determine that miR-145 directly binds to and negatively regulates OPG expression. Notably, when the miR-145 binding site in the reporter vector carrying the OPG 3'-UTR was mutated, the stimulation of estrogen on the reporter activity was not completely abolished (data not shown). It is common for 3'-UTRs in mRNA to contain several miRNA-binding sites; therefore, additional miRNAs that may be involved in this process cannot be excluded. It has previously been reported that the expression levels of numerous miRNAs are altered following estrogen treatment $(32,33)$. These estrogen-regulated miRNAs may be involved in regulating OPG expression in a direct manner or indirect manner by targeting OPG regulators. Further investigation is required to examine the action of these miRNAs on OPG expression and the underlying mechanism.

In conclusion, the present study indicated an indirect regulation of estrogen on OPG expression via modulating miR-145 expression. Estrogen suppressed miR-145 expression, and OPG was confirmed to be a direct target of miR-145. Decreased miR-145 expression, as induced by estrogen, resulted in the release of OPG 3'-UTR, thus reviving OPG expression. These findings may extend the understanding regarding the molecular mechanisms underlying estrogen-mediated OPG induction.

\section{References}

1. Rachner TD, Khosla S and Hofbauer LC: Osteoporosis: Now and the future. Lancet 377: 1276-1287, 2011.

2. Venken K, Callewaert F, Boonen S and Vanderschueren D: Sex hormones, their receptors and bone health. Osteoporos Int 19: $1517-1525,2008$

3. Makras P, Delaroudis S and Anastasilakis AD: Novel therapies for osteoporosis. Metabolism 64: 1199-1214, 2015.

4. Lindberg MK, Erlandsson M, Alatalo SL, Windahl S, Andersson G, Halleen JM, Carlsten H, Gustafsson JA and Ohlsson C: Estrogen receptor alpha, but not estrogen receptor beta, is involved in the regulation of the OPG/RANKL (osteoprotegerin/receptor activator of NF-kappa B ligand) ratio and serum interleukin-6 in male mice. J Endocrinol 171: 425-433, 2001.

5. Boyce BF and Xing L: Functions of RANKL/RANK/OPG in bone modeling and remodeling. Arch Biochem Biophys 473: 139-146, 2008.

6. Silva I and Branco JC: Rank/Rankl/opg: Literature review. Acta Reumatol Port 36: 209-218, 2011.

7. Lacey DL, Boyle WJ, Simonet WS, Kostenuik PJ, Dougall WC, Sullivan JK, San Martin J and Dansey R: Bench to bedside: Elucidation of the OPG-RANK-RANKL pathway and the development of denosumab. Nat Rev Drug Discov 11: 401-419, 2012.

8. Eghbali-Fatourechi G, Khosla S, Sanyal A, Boyle WJ, Lacey DL and Riggs BL: Role of RANK ligand in mediating increased bone resorption in early postmenopausal women. J Clin Invest 111: 1221-1230, 2003

9. Woo KM, Choi Y, Ko SH, Ko JS, Oh KO and Kim KK: Osteoprotegerin is present on the membrane of osteoclasts isolated from mouse long bones. Exp Mol Med 34: 347-352, 2002.

10. Kondo T, Kitazawa R, Maeda S and Kitazawa S: 1 Alpha, 25 dihydroxyvitamin D3 rapidly regulates the mouse osteoprotegerin gene through dual pathways. J Bone Miner Res 19: 1411-1419, 2004.

11. Anderson DM, Maraskovsky E, Billingsley WL, Dougall WC, Tometsko ME, Roux ER, Teepe MC, DuBose RF, Cosman D and Galibert L: A homologue of the TNF receptor and its ligand enhance T-cell growth and dendritic-cell function. Nature 390: 175-179, 1997.

12. Hsu H, Lacey DL, Dunstan CR, Solovyev I, Colombero A, Timms E, Tan HL, Elliott G, Kelley MJ, Sarosi I, et al: Tumor necrosis factor receptor family member RANK mediates osteoclast differentiation and activation induced by osteoprotegerin ligand. Proc Natl Acad Sci USA 96: 3540-3545, 1999.

13. Hofbauer LC, Khosla S, Dunstan CR, Lacey DL, Spelsberg TC and Riggs BL: Estrogen stimulates gene expression and protein production of osteoprotegerin in human osteoblastic cells. Endocrinology 140: 4367-4370, 1999.

14. Bord S, Ireland DC, Beavan SR and Compston JE: The effects of estrogen on osteoprotegerin, RANKL, and estrogen receptor expression in human osteoblasts. Bone 32: 136-141, 2003.

15. Saika M, Inoue D, Kido S and Matsumoto T: 17beta-estradiol stimulates expression of osteoprotegerin by a mouse stromal cell line, ST-2, via estrogen receptor-alpha. Endocrinology 142: 2205-2212, 2001

16. Manokawinchoke J, Ritprajak P, Osathanon T and Pavasant P: Estradiol induces osteoprotegerin expression by human dental pulp cells. Odontology 104: 10-18, 2016.

17. Bekker PJ, Holloway D, Nakanishi A, Arrighi M, Leese PT and Dunstan CR: The effect of a single dose of osteoprotegerin in postmenopausal women. J Bone Miner Res 16: 348-360, 2001.

18. Michael H, Härkönen PL, Väänänen HK and Hentunen TA: Estrogen and testosterone use different cellular pathways to inhibit osteoclastogenesis and bone resorption. J Bone Miner Res 20: 2224-2232, 2005.

19. Rumpler M, Varga F and Nemeth P: Identification of an estrogen response element in the osteoprotegerin promoter. Bone 33: S17.

20. Bartel DP: MicroRNAs: Genomics, biogenesis, mechanism, and function. Cell 116: 287-297, 2004.

21. Hassan MQ, Tye CE, Stein GS and Lian JB: Non-coding RNAs: Epigenetic regulators of bone development and homeostasis. Bone 81: 746-756, 2015.

22. Jia J, Tian Q, Ling S, Liu Y, Yang S and Shao Z: MiR-145 suppresses osteogenic differentiation by targeting Sp7. FEBS Lett 587: 3027-3031, 2013. 
23. Fukuda T, Ochi H, Sunamura S, Haiden A, Bando W, Inose $H$, Okawa A, Asou Y and Takeda S: MicroRNA-145 regulates osteoblastic differentiation by targeting the transcription factor Cbfb. FEBS Lett 589: 3302-3308, 2015.

24. Yang N, Wang G, Hu C, Shi Y, Liao L, Shi S, Cai Y, Cheng S, Wang X, Liu Y, et al: Tumor necrosis factor $\alpha$ suppresses the mesenchymal stem cell osteogenesis promoter miR-21 in estrogen deficiency-induced osteoporosis. J Bone Miner Res 28 : 559-573, 2013.

25. Chen C, Cheng P, Xie H, Zhou HD, Wu XP, Liao EY and Luo XH: MiR-503 regulates osteoclastogenesis via targeting RANK. J Bone Miner Res 29: 338-347, 2014.

26. Pitari MR, Rossi M, Amodio N, Botta C, Morelli E, Federico C, Gullà A, Caracciolo D, Di Martino MT, Arbitrio M, et al: Inhibition of miR-21 restores RANKL/OPG ratio in multiple myeloma-derived bone marrow stromal cells and impairs the resorbing activity of mature osteoclasts. Oncotarget 6: 27343-27358, 2015.

27. Livak KJ and Schmittgen TD: Analysis of relative gene expression data using real-time quantitative PCR and the 2(-Delta Delta C(T)) Method. Methods 25: 402-408, 2001.

28. Prossnitz ER, Arterburn JB, Smith HO, Oprea TI, Sklar LA and Hathaway HJ: Estrogen signaling through the transmembrane G protein-coupled receptor GPR30. Annu Rev Physiol 70: 165-190, 2008.

29. Bashir A, Mak YT, Sankaralingam S, Cheung J, McGowan NW, Grigoriadis AE, Fogelman I and Hampson G: Changes in RANKL/OPG/RANK gene expression in peripheral mononuclear cells following treatment with estrogen or raloxifene. Steroids 70: 847-855, 2005.
30. Rachner TD, Schoppet M, Niebergall U and Hofbauer LC: 17beta-Estradiol inhibits osteoprotegerin production by the estrogen receptor-alpha-positive human breast cancer cell line MCF-7. Biochem Biophys Res Commun 368: 736-741, 2008.

31. Castellano L, Giamas G, Jacob J, Coombes RC, Lucchesi W, Thiruchelvam P, Barton G, Jiao LR, Wait R, Waxman J, et al: The estrogen receptor-alpha-induced microRNA signature regulates itself and its transcriptional response. Proc Natl Acad Sci USA 106: 15732-15737, 2009.

32. Klinge CM: Estrogen regulation of MicroRNA expression. Curr Genomics 10: 169-183, 2009.

33. Klinge CM: miRNAs regulated by estrogens, tamoxifen, and endocrine disruptors and their downstream gene targets. Mol Cell Endocrinol 418: 273-297, 2015.

34. Sachdeva M and Mo YY: miR-145-mediated suppression of cell growth, invasion and metastasis. Am J Transl Res 2: 170-180, 2010.

35. Hu H, Zhang Y, Cai XH, Huang JF and Cai L: Changes in microRNA expression in the MG-63 osteosarcoma cell line compared with osteoblasts. Oncol Lett 4: 1037-1042, 2012.

36. Li E, Zhang J, Yuan T and Ma B: MiR-145 inhibits osteosarcoma cells proliferation and invasion by targeting ROCK1. Tumour Biol 35: 7645-7650, 2014. 